\title{
Classification of epilepsy types through global network analysis of scalp electroencephalograms
}

\author{
UnCheol Lee* and Seunghwan Kim ${ }^{\dagger}$ \\ Asia Pacific Center for Theoretical Physics \& Nonlinear Complex Systems Laboratory, National Core Research Center \\ on System Biodynamics, Department of Physics, Pohang University of Science and Technology, Pohang, 790-784, Korea \\ Ki-Young Jung ${ }^{\ddagger}$ \\ Department of Neurology, Samsung Medical Center, Sungkyungkwan University School of Medicine, 50 Ilwon-dong, Gangnam-Gu, \\ Seoul, 135-710 Korea \\ (Received 17 May 2005; revised manuscript received 28 October 2005; published 17 April 2006)
}

\begin{abstract}
Epilepsy is a dynamic disease in which self-organization and emergent structures occur dynamically at multiple levels of neuronal integration. Therefore, the transient relationship within multichannel electroencephalograms (EEGs) is crucial for understanding epileptic processes. In this paper, we show that the global relationship within multichannel EEGs provides us with more useful information in classifying two different epilepsy types than pairwise relationships such as cross correlation. To demonstrate this, we determine the global network structure within channels of the scalp EEG based on the minimum spanning tree method. The topological dissimilarity of the network structures from different types of temporal lobe epilepsy is described in the form of the divergence rate and is computed for 11 patients with left (LTLE) and right temporal lobe epilepsy (RTLE). We find that patients with LTLE and RTLE exhibit different large scale network structures, which emerge at the epoch immediately before the seizure onset, not in the preceding epochs. Our results suggest that patients with the two different epilepsy types display distinct large scale dynamical networks with characteristic epileptic network structures.
\end{abstract}

DOI: 10.1103/PhysRevE.73.041920 PACS number(s): 87.19.La, 87.90.+y, 89.75.-k, 87.19.Xx

\section{INTRODUCTION}

Until now, the relationships between multichannel sites of the electroencephalogram (EEG) have been one of the most interesting subjects of research. To understand their complex and dynamic relationships, various methods such as interdependence, synchronization and information flow between multichannels have been investigated [1-6]. In the previous works it was reported that an epileptogenic site, a site having the capacity to induce epilepsy, or a hemisphere that contains a seizure focus, show different characteristics for various nonlinear measures. Based on findings in animal models of epilepsy, Lehnertz et al. hypothesized that neuronal networks involved in the epileptic process exhibit a decreased level of complexity even during the interictal state. In the EEG the recording during an actual seizure is said to be ictal and interictal refers to the state between ictal events. The measure of the neuronal complexity loss unequivocally lateralizes and often localizes the primary epileptogenic area even without recording transients such as seizures or spikes $[1,2]$. In the investigation of the degree of interdependence between EEG channels, the transient patterns of nonlinear interdependency emerge at the initial spread of the seizure during the essential phase of its development and at the end of the seizure. However, the maintenance of these interactions has not been observed throughout the seizure activity. From the investigation of the interdependency, it is proposed that

\footnotetext{
*Electronic address: uclee@ postech.ac.kr

†Electronic address: swan@ postech.ac.kr

${ }^{\ddagger}$ Electronic address: kyjung@smc.samsung.ac.kr
}

nonlinear associations play an important role in epileptogenics, and that the process of the neuronal entrainment during the seizure onset involves a transient interaction between a distributed network of neuronal aggregates [3]. The hemisphere containing the epileptogenic focus is characterized by the increase of phase synchronization during the interictal period and the onset of epileptic seizures is preceded by the decrease in phase synchronization during the preictal period $[5,6]$. These works suggest that epilepsy is a dynamic disease in which the neural activity and the interrelationship between neurons or the local neuronal networks in the brain changes transiently from the interictal to the ictal state through the preictal state. Moreover, such characteristic changes are directly associated with the epileptogenic site and the hemisphere containing the focal site.

Recently, in cognitive sciences, the large scale integration network has been the subject of great interest. At the moment of perception, large-scale integration occurs between different areas on the scalp such as frontal, parietal, and occipital areas, with a significant increase in the $\gamma$ wave. Neural phase synchrony is an important candidate for such a large-scale integration that is mediated by neural groups oscillating in specific bands and entering into precise phase locking over a transient period of time. Such synchronous patterns are continuously created, destroyed, and subsequently recreated on multiple spatial and temporal scales in the nervous system [7-9]. These phenomena of the emergent large-scale integration of the scalp EEG are related primarily to various brain functions such as perception, consciousness, and visual or auditory sensation.

These clinical manifestations of seizures are connected to the area of the cortex in which the seizures start, and how widely they propagate and how long they last. A local epi- 
leptic activity often creates experiential events in the mind of the patient. Typically, experiential phenomena occur at the beginning of the seizure and are a part of the patient's aura. Based on the these facts, Le Van Quyen et al. assumed that there may exist a large-scale epilepsy network [10]. In fact, the survey data from recent research on seizures and behavior shows that $63-92 \%$ of people with epilepsy identify situational precipitants [11]. Furthermore, in one study, it was found that the mood changes significantly in the days before and after seizures [12]. The identification of an impending seizure and the change of the mood of patients a few days ahead correspond to the preictal state, which has been found in some nonlinear studies. These results suggest that the procedure of epileptic seizure is connected to the experience of the patients and the experience constructs a largescale epilepsy network in the brain.

We assume that in the impending epoch before the seizure onset, a certain type of network connected to the seizure is activated within the cortex, whose dynamical structure might be different in various epilepsy types with different focal sites. In order to investigate the network structure of epileptic EEGs, we choose two representative types of epilepsy, the left (LTLE) and the right temporal lobe epilepsy (RTLE) with the epileptogenic focus on the opposite sides of the brain. First, we investigate the pairwise relationship with the correlation between multichannels and its time evolution. In the next section, for more detailed investigation of the network structure of EEGs, we construct a hierarchical network structure for multichannel scalp EEGs which may be useful to understand how the channels form a global network. Therefore, we define the channel network of the scalp EEG based on the minimum spanning tree (MST), which provides a unique network structure for the scalp EEG data and enables us to define a global channel relationship for EEGs from a patient. From the constructed channel networks, the dissimilarities between the channel networks are computed for 11 epilepsy patients (six LTLE and five RTLE patients).

To quantify the similarity between two network structures, we define a nonlinear measure, called the divergence rate of the MST, $D_{(X, Y)}$, which is associated with the normalized tree length and the survival ratio, originally introduced by Onnela et al. [13-15] to investigate the dynamic change of the asset tree for S\&P 500 companies. The measure $D_{(X, Y)}$ quantifies how similar two network structures are. We computed $D_{(X, Y)}$ for the channel networks for all patients, whose similarities are classified into two different epilepsy types with the help of the dendrogram method. Furthermore, the time evolution of $D_{(X, Y)}$ is studied for an EEG time series $40 \mathrm{~min}$ long and with one end at the seizure onset that is segmented into five epochs with 8 min time intervals.

The dendrogram for each segmented EEG epoch shows that the network structures for LTLE and RTLE groups, which cannot be distinguished in the beginning epoch, can be distinguished during the epoch preceding the seizure onset. These two groups can be classified quite well around the $1 \mathrm{~min}$ epoch, just before the seizure onset, suggesting the appearance of two classes of network structures for two different epilepsy types. In particular, the network structures of the LTLE group show stronger similarities, indicating the emergence of a characteristic large-scale epilepsy network for the LTLE patients before the seizure onset which may help to facilitate the ensuing seizure onset.

\section{METHOD AND APPLICATION}

\section{A. Pairwise relationship of multichannel EEGs}

In this section, we study the pairwise relationship of multichannel EEGs with the help of cross correlation. We acquired the scalp EEGs from 11 patients, who are clinically well classified into two epilepsy types, left and right temporal lobe epilepsy. Scalp EEG data were obtained during video-EEG monitoring from the patients with medically intractable temporal lobe epilepsy who were candidates for surgical treatment. The epileptogenic focus was determined by presurgical evaluation including long-term video-EEG monitoring, interictal and ictal SPECT, volumetric magnetic resonance imaging MRI, neuropsychological tests, and ${ }^{18}$ F-fluorodeoxyglucose (FDG) PET, if necessary. The epileptogenic focuses are located on left lateral (four subjects), left mesial (two subjects), and right mesial (five subjects). A digital EEG machine (Vanguard system, Cleveland Clinic Foundation Healthcare Ventures, Inc., Ohio) was used to record the EEGs from 43 electrodes of the 10-20 system with sphenoidal electrodes referenced to Pz. The electrode impedance was maintained at less than $5 \mathrm{k} \Omega$. Filter settings were $0.5-70 \mathrm{~Hz}$, with a sampling rate of $200 \mathrm{~Hz}$ and 12-bit analog-to-digital precision.

The total recording time is about $40 \mathrm{~min}$ before the seizure onset. In order to investigate the time evolution of correlations between channels and the difference between two hemispheres, the 40-min-long EEG data was segmented into five windows of $8 \mathrm{~min}$, and a $1 \mathrm{~min}$ noise-free EEG epoch is extracted from each window. We can extract the noise-free EEG epochs from all EEG channels during 1 min before the seizure onset and during at least $1 \mathrm{~min}$ at each segmented window from only 11 out of 50 patients. Since the EEG could be unexpectedly contaminated by severe artifacts, we choose the clean EEG epochs from the EEG data to avoid any ambiguity of the result, albeit with fewer statistics. One minute in EEG recording is sufficiently long for representing the other $7 \mathrm{~min}$ in the window and it could reflect the patient's state at the EEG epoch. The minimal statistics are obtained by dividing the 1 min interval into six $10 \mathrm{~s}$ subintervals.

First, we calculate the cross correlation between the 43 channels of the EEG in order to study the pairwise relationship of multichannel EEGs for 11 epilepsy patients. We acquire the time series of a channel $i, r_{i}=x_{1}, x_{2}, \ldots, x_{M}$, where $M$ is the total length of a sequence. The cross correlation between channel $i$ and $j$ is defined as follows:

$$
C_{i j}=\frac{\left\langle r_{i} r_{j}\right\rangle-\left\langle r_{i}\right\rangle\left\langle r_{j}\right\rangle}{\sqrt{\left[\left\langle r_{i}^{2}\right\rangle-\left\langle r_{j}\right\rangle^{2}\right]\left[\left\langle r_{i}^{2}\right\rangle-\left\langle r_{j}\right\rangle^{2}\right]}}
$$

where $\langle\cdots\rangle$ indicates a time average over the 1 min epoch. The 1 min EEG epochs selected from 11 patients are used to calculate the mean cross correlation at each window for each patient. The mean cross correlation is defined by $\overline{C(i, j)}$ 
$=[2 / n(n-1)] \sum_{i}^{n} \sum_{j(j<i)}^{n} C_{i j}, i, j=1, \ldots, n$, where the number of channels $n$ is 43 .

We separate the EEG data set into two epilepsy types (six LTLE and five RTLE) for which the mean cross correlation is computed as in Fig. 1. In order to study the difference between both types of epilepsy and the time variation within a group, we used a repeated measures analysis of variance (ANOVA) using the generalized linear model (GLM). The criterion for statistical significance is $p<0.05$. The test shows that the interaction between the type of epilepsy and the time window is not significant $[F(4,36)=1.86 ; P$ $=0.139>0.05]$ and no significant effect on the type of epilepsy exists $[F(1,9)=0.05 ; P=0.8212>0.05]$. But the time window within a group has a significant effect $[F(4,36)$ $=3.16 ; P=0.025<0.05]$. All data were analyzed with SAS statistical tools.

The global features of the pairwise channel relationships are shown in Figs. 1(b) and 1(c). Roughly, the two epilepsy groups exhibit different global features of the channel relationship. In particular, the left frontal channels (LFs) of the LTLE group have a relatively strong correlation with the channels of the other areas. From this, we could estimate that there exists a characteristic global network structure depending on the epilepsy types. Instead of the pairwise channel relationship, a more detailed investigation of the global channel relationship may provide additional insight into the two epilepsy groups. In order to investigate the differences in the channel relationship between two different types of epilepsy, we construct a hierarchical network structure for multichannel scalp EEGs which may be useful for providing us with information about the spatial or functional clustering of EEG channels and understanding how a channel and a subcluster of local channels are linked to other channels or subclusters and how such subclusters form a global network. In the next section, we will introduce the method for constructing a hierarchical network structure of EEG channels and comparing such structures reliably in classifying different epilepsy types.

\section{B. Global relationship of multichannel EEG (minimum spanning tree)}

As a simple method to define the multichannel relationship, we use the minimum spanning tree, which is a well known method for finding the shortest path between nodes in the graph theory. Since the EEGs of different channels are distinct and highly nonstationary, the MST produces a distinct network structure for a given multi-channel EEG data. In this paper, we construct a network structure based on the MST from 43 channel EEG data and define the relationship between all channels of a patient based on this network structure. In most previous works, the relationship between channels of a patient was not uniquely determined because it depends significantly on the subjective parameters such as the cutoff threshold. The MST method has an advantage because it allows us to construct a unique network without any subjective parameters and compare two different network structures systematically.

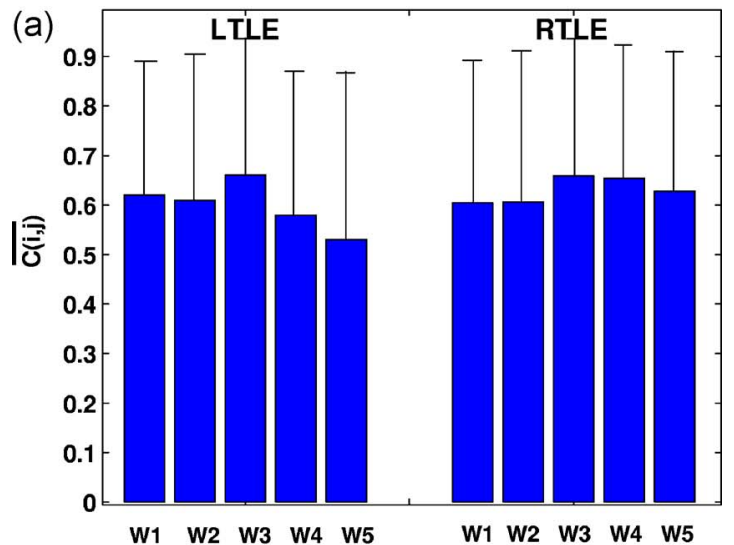

(b)
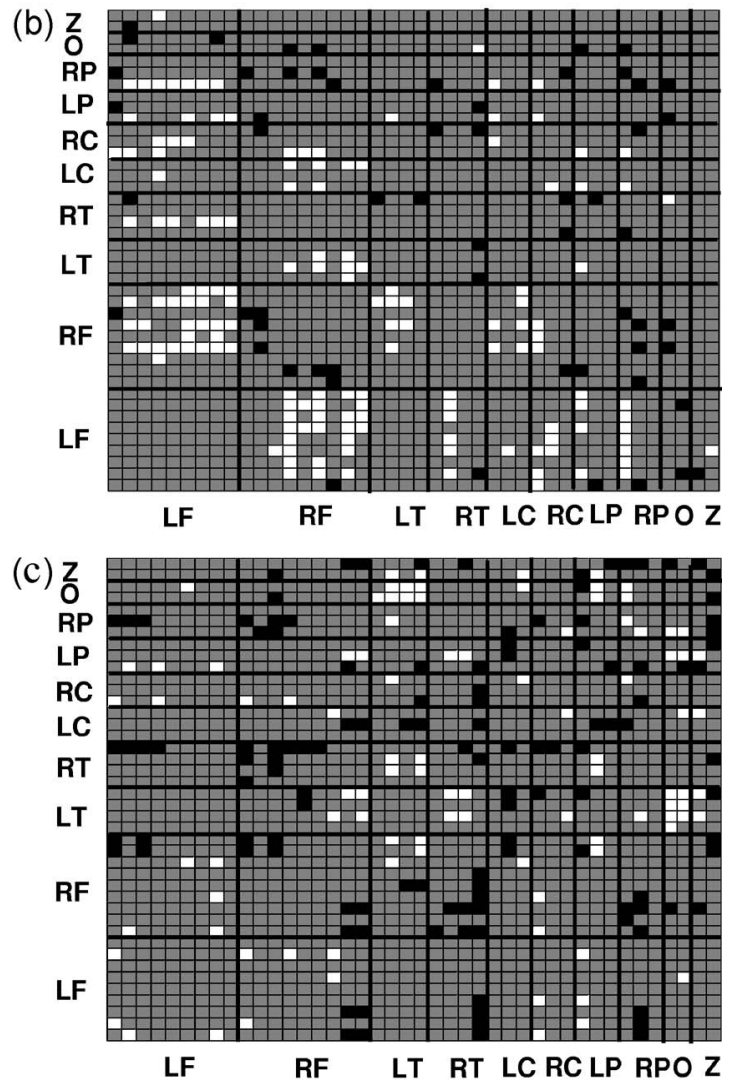

FIG. 1. (Color online) The mean cross correlations between channels, $\overline{C(i, j)}$, and their standard deviations are calculated for two types of epilepsy patients (six with LTLE and five with RTLE), which are averaged over the five segmented EEG periods. (a) The time evolution of the mean cross correlations in two epilepsy groups (six with LTLE and five with RTLE) with the standard deviation bar. (b), (c) The average difference of $\overline{C(i, j)}$ between window 4 and window 5, in LTLE and RTLE groups, respectively. The white indicates the pair of channels above the upper $10 \%$, while the black indicates those below the lower 10\%. [LF (left frontal): FP1,F7,F3,AF7,F5,FC5,FT7,F9,FT9. RF (right frontal): Fp2,F8,F4,AF8,F6,FC6,FT8,F10,FT10. LT (left temporal): T7,TP7,T9,TP9. RT (right temporal): T7,TP8,T10,TP10. LC (left central): C3,C5,CP5. RC (right central): C4,C6,CP6. LP (left parietal): P7,P3,P9. RP (right parietal): P4,P8,P10. O (occipital): O1,O2. Z(zero line): $\mathrm{Cz}, \mathrm{Pz}]$. 
In order to construct the MST, first we define a distance matrix based on the correlation coefficients in Eq. (1). From $N$ channels used to construct the MST, we compute an $N$ $\times N$ matrix of correlation coefficients $-1 \leqslant C_{i j} \leqslant 1$, which can be transformed into an $N \times N$ distance matrix with elements $d_{i j}=\sqrt{2\left(1-C_{i j}\right)}$, such that $\sqrt{2} \geqslant d_{i j} \geqslant 0$, respectively. The elements $d_{i j}$ satisfy the axioms for the metric distance, (i) $d_{i j}=0$ if and only if $i=j$, (ii) $d_{i j}=d_{j i}$, (iii) $d_{i j} \leqslant d_{i k}+d_{k j}$, and those for ultrametricity. The distance matrix can be used to determine the minimum spanning tree based on the distances, which is a simply connected graph that connects all the $N$ nodes of the graph with $N-1$ edges such that the sum of all edge weights, $\sum_{(i, j) \in r} d_{i j}$ is a minimum. Note that in this connection a loop is not allowed.

The MST effectively reduces the information space from $N(N-1) / 2$ correlation coefficients to $N-1$ tree edges. Such a construction of the MST has proven to be useful in diverse applications due to its simplicity and uniqueness [16-18]. It also allows us to easily visualize the relationship between all channels in the form of the tree. Based on this construction, we can easily track the change in the tree structure, in particular, the evolution of a specific channel in the interrelationship during the dynamical change from an interictal state to an ictal state. To quantify how much two network structures are similar, we define a modification of the measure, the survival ratio $\sigma(a, b)$, which was originally introduced by Onnela et al. [13]. The survival ratio quantifies how many directly linked nodes in a MST remain in comparison, which has been previously applied to the investigation of the dynamic change of the asset tree of S\&P 500 companies:

$$
\sigma(a, b)=\frac{1}{N-1}\left|E_{a} \cap E_{b}\right|
$$

where $E_{a}$ and $E_{b}$ refer to the set of edges of two MSTs $a$ and $b$, respectively, $\cap$ is the intersection operator, and $|\cdots|$ gives the number of elements in the set. In other words, the survival ratio is the fraction of edges found in common in both graphs, which was originally used to study the time evolution of tree structures. Note that the survival ratio just compares the linkage of nodes in two graphs. In dynamically changing graphs such as ones for an epileptic process, the distance between edges can expand or shrink due to the enhancement or the reduction in correlation between channels on a local scalp area. This leads us to define a measure which can take into account of the change in both distance and the linkage of nodes in a graph at the same time.

\section{Dissimilarity measure for minimum spanning trees}

To quantify the similarity or the dissimilarity between two MSTs, we define a measure based on the information theory. In order to compare and classify the MSTs constructed, the measure should be a metric which satisfies the metric properties. Crutchfield developed the information metric $d(X, Y)$ between two information sources $X$ and $Y$ in the information space $I[19]$,

$$
d(X, Y) \equiv H(X \mid Y)+H(Y \mid X)
$$

where $H(X \mid Y)$ and $H(Y \mid X)$ are the conditional entropies between information sources $X$ and $Y$. This information metric $d(X, Y)$ satisfies three axioms: $d(X, Y)=d(Y, X), d(X, Y)=0$ if and only if $X \cong Y, d(X, Z) \leqslant d(X, Y)+d(Y, Z)$, and it is a measure of the noncommonality or the difference between $X$ and $Y$. Therefore, to apply this information metric concept to our case, the distance matrix $T$ of a MST is considered as the information source, which contains the information about the linkage and the size of edges of all $N$ nodes. To determine the distance between two MSTs $X$ and $Y$ we calculate $H(X \mid Y)+H(Y \mid X)$. The conditional entropy $H(X \mid Y)$ specifies the amount of information that is gained by measuring an information source $Y$ with the knowledge of another $X$, which can be approximated by the information change between two different information sources, $X$ and $Y$.

The information source $X$ is transformed into the information source $Y$ by $f: X \rightarrow Y$. Suppose that the information source $X$ is contaminated with the uncorrelated noise, so that there is some uncertainty $\Delta x_{i}$. The uncertainty after the transformation $f$ is given by $\left|f\left(\Delta x_{i}\right)=\Delta f\left(x_{i}\right)\right|$. Thus, the information change by the transformation $f$ is $\ln \left|\frac{\Delta f\left(x_{i}\right)}{\Delta x_{i}}\right|$. The ratio $\left|\frac{\Delta f\left(x_{i}\right)}{\Delta x_{i}}\right|$ is the amplification of an initial uncertainty after the transformation. The average information change is defined as

$$
\overline{\Delta H}=\frac{1}{N} \sum_{i=1}^{N} \ln \left|\frac{\Delta f\left(x_{i}\right)}{\Delta x_{i}}\right|,
$$

where $N$ is the number of elements of an information source. The average information change also quantifies the amount of information gain by the transformation of the information source, $Y=f(X)$, given an information source $X$. This average information change between two information sources corresponds to the conditional entropy, $H(X \mid Y)=H(X, Y)-H(X)$. In practice, in order to apply the average information change between two MSTs, we calculate the divergence rate of two MSTs, $D_{(Y \mid X)}$, as an approximation,

$$
D_{(Y \mid X)}=\frac{1}{N} \sum_{i=1}^{N} \log _{10}\left|\frac{D_{(Y \mid X)(i)}}{D_{X(i)}}\right|,
$$

where $D_{X(i)}$ is the sum of all distances taken from a reference node $i$ to the neighborhood nodes $i_{n n}$ in the $X_{M S T}$, and $D_{(Y \mid X)(i)}$ is the sum of all distances taken from the node $i$ to the nodes $i_{n n}$ in the $Y_{M S T}$. Given the distances between a node $i$ and its neighbor nodes in the $X_{M S T}, D_{(Y \mid X)(i)}$ evaluates how much their distance change in the $Y_{M S T}$. Each distance is defined as $D_{Z(i)}=\sum_{i_{n n}=1}^{N_{n}(i)} d_{i_{n n}}, d_{i_{n n}}=\operatorname{dist}\left(\operatorname{Node}_{i}, \operatorname{Node}_{i_{n n}}\right)$, where the function $\operatorname{dist}(\cdots)$ gets the sum of all distances on the path traversed from node $i$ to node $i_{n n}$. Hence, $N_{n}(i)$ is the number of neighbors of the node $i$, which varies for each reference node, depending on its location on the tree structure. Therefore, $D_{(Y \mid X)}$ approximates the conditional entropy of two distance matrices of two MSTs, quantifying how much information is needed on average to explain $Y_{M S T}$, given $X_{M S T}$. Since 

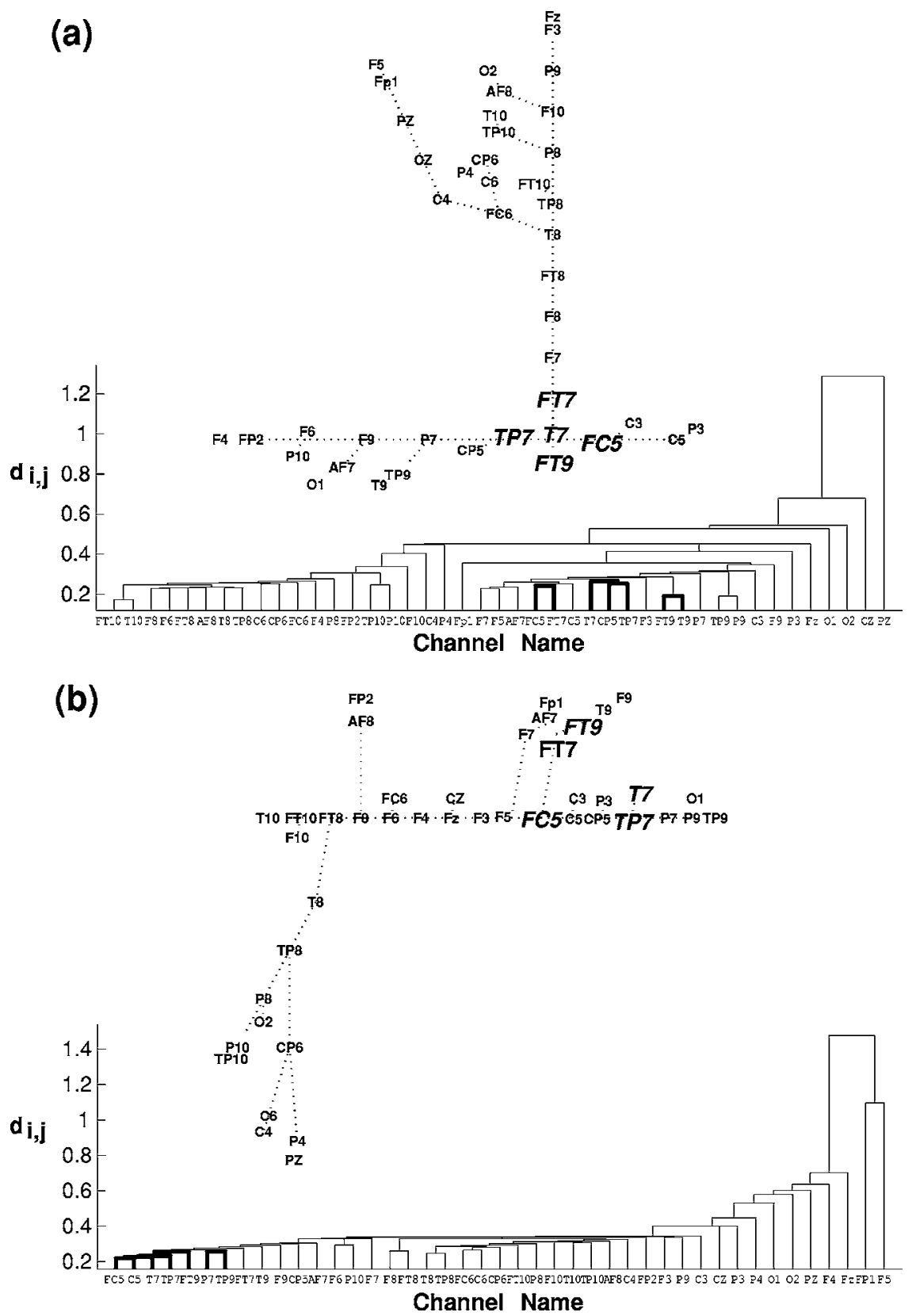

FIG. 2. (a) MST of a LTLE patient. (b) MST of a RTLE patient. $D_{(X, Y)}$ quantifies how far the neighborhood channels TP7, FT7, FC5, and FT9 of the T7 in the MST of a LTLE are located in the MST of a RTLE patient. The subplots are the dendrograms of the 43 EEG channels. The bold lines indicate the distance between the neighborhood channels and the reference channel. The MST gives information on the linkage of channels, while the dendrogram gives information on the distances between the channels.

$D_{(Y \mid X)}$ and $D_{(X \mid Y)}$ are asymmetric, we define the metric distance between two MSTs as follows:

$$
D_{(X, Y)}=D_{(Y \mid X)}+D_{(X \mid Y)} .
$$

The distance $D_{(X, Y)}$ quantifies the dissimilarity between two MST structures. If two network structures are exactly the same, $D_{(X, Y)}$ is 0 . Otherwise, $D_{(X, Y)}$ is larger than 0 . Since the information sources, $X_{M S T}$ and $Y_{M S T}$ contain the information about the linkage and the size of the edge of nodes, the dissimilarity between two information sources can reflect the difference between two configurations of MST's in a more general way.

Figure 2 shows where neighboring channels (TP7, FT7, FC5, FT9) of the channel T7 in the network of a LTLE patient [Fig. 2(a)] are located in the network of a RTLE patient [Fig. 2(b)]. We quantify how far these neighborhood channels are located from a reference channel T7 in a RTLE network. In this example, TP7, FT7, FC5, and FT9, are 1, 5, 4 , and 6 steps apart from the reference channel T7, respectively. The sum of all distances from the reference channel T7 for all neighboring channels is computed and then compared by summing all distances in the LTLE network. This process is repeated for all channels (nodes) to compute $D_{(X, Y)}$.

\section{Classification of two different epilepsy groups based on $D_{(X, Y)}$}

We apply $D_{(X, Y)}$ to MSTs constructed from the scalp EEGs of 11 epilepsy patients for the classification of two epilepsy types. In this work, by comparing the similarities of their tree structure, we attempt to find whether there exists any typical 
tree structure for two epilepsy types and when such typical tree structures appear during the epileptic transition process. In order to do this, we calculate $D_{(X, Y)}$ for all pairs of 11 MSTs constructed from a segmented EEG period, calculating the $11 \times 11 D_{(X, Y)}$ matrices from the five segmented EEG periods. Since $D_{(X, Y)}$ measures the dissimilarity of MSTs, we use the dendrogram for a simple hierarchical clustering of MSTs. From this, dendrograms for five segmented EEG periods can be computed and compared during the epileptic process. In order to construct a dendrogram, we need to identify two MSTs with the most similarity, that is, the smallest $D_{(X, Y)}$. This pair builds the first cluster. In the next step, a pair can be formed by joining either two MSTs, or a MST and the cluster of MST. The process is then repeated iteratively, by forming a cluster from the pair of closest MSTs or clusters of MSTs, until all of them are connected to form a rooted tree [20]. Figure 3 presents the result of clustering of MSTs by the dendrogram method. From the dendrogram constructed in each segmented EEG epochs, we found that in the preceding epochs (a), (b), (c), and (d), the two types of epilepsy cannot be classified well. In the epoch (e), corresponding to 1 min before the seizure onset, however, the two groups are classified quite well. In particular, by setting an appropriate threshold, we can classify these two groups, except for only one patient who is falsely classified into the RTLE group. This emergence of the clear classification suggests that the network structures involved in either group become similar before the seizure onset, that is, typical network structures emerge for these two types of epilepsy. The time evolution of the network shows that such tendency in classification already appears in the epochs (c) and (d), in which a few network structures in LTLE or RTLE groups become clustered as a subgroup. The similarity in network structures begins to occur even in the preceding epoch during two epochs before the onset of the seizure. We find that the occurrence of a similar network structure is strongly correlated with the occurrence of the preictal state, which has been reported in the previous work on seizure anticipation. The duration and strength of the epileptic state of a patient depends much on the individuals. In particular, the duration varies widely from a few minutes to a few hours [21-24]. These individual differences in the epileptic processes make it difficult to extrapolate the classification of two epilepsy groups in the earlier epochs. However, at one minute before the seizure onset, most patients would be faced with the preictal state, so that we could estimate the classification of two epilepsy types at the EEG period.

As expected, the LTLE epilepsy group is well clustered by $D_{(X, Y)}$ in Fig. 3(e), except for one patient, during the EEG period before the seizure onset. In comparison, the classification attempt by the survival ratio in Fig. 3(f) is not so successful. The significant difference in the clustering performance for two methods is due to the fact that the survival ratio considers only the linkage of nodes of two graphs in comparison, while $D_{(X, Y)}$ considers both the linkage of nodes and the distance of edges, simultaneously. Therefore, $D_{(X, Y)}$ reflects the shrink or expansion of edges in a local subtree of the EEG channel networks quite well, which result from the change in correlation between some channels on the left or the right hemispheres of an epilepsy patient. We showed that the mean cross correlation of channels can vary depending on hemispheres and EEG epochs. Note that under the weak noisy environment the linkage of some nodes in a graph could be distorted and misplaced, which can be better handled by $D_{(X, Y)}$ with the distance metric. To investigate the robustness of the classification in Fig. 3(e), we segment the fifth EEG epoch into six EEG intervals of $10 \mathrm{~s}$ long and construct the dendrograms in the EEG segments. We find that the all six dendrograms have the same hierarchical clustering structure. In Figs. 4(a)-4(f), five LTLE patients and four RTLE patients are classified as LTLE and RTLE groups, respectively, and the two patients, one LTLE and one RTLE patient, are classified as another group and those hierarchical clustering structures are sustained during the six segmented EEG intervals. This implies that the classification of the two types of epilepsy based on the global network of the scalp EEG channels is also robust even in the $10 \mathrm{~s}$ EEG time scale. The difference between the dendrograms constructed in the different EEG time scales could occur due to the lower frequency or higher frequency dominance in the different EEG time scales. Further investigation on the dependence of the global network structure on the EEG time scale is needed.

There are some common channel connections in each type of patients but those channel connections do not provide any information about lateralization because they are globally distributed on both hemispheres. Therefore, the pairwise relationship of specific scalp EEG channels is not appropriate for obtaining any meaningful information because the local brain functions and their functional relationship changes all the time due to their perpetual internal and external activities. Instead, the global feature of the channel relationship provides us with relatively robust and meaningful information on the brain state of the epilepsy patient, in spite of frequent local fluctuations in its network structure. Our results suggest that a seizure focus located on one hemisphere recruits the channels around it, constructing a latent characteristic network. With a trigger turned on, this latent network surfaces, facilitating a seizure onset. This assumption for the existence of a latent network might be useful for explaining the dynamic characteristic of the brain of the epilepsy patient.

In order to test the robustness of the classification of two types of epilepsy, we applied a perturbation test with weighted average discrepancy pairs (WADP) [25]. The WADP method evaluates the robustness of the clustering result by comparing the clustering of the original data set and the perturbed data set. The perturbed EEG of a channel $i\left(y_{i}\right)$ is defined as $y_{i}=x_{i}+R(k) s\left(x_{t}\right) g_{i}$, where $x_{i}$ is the normalized EEG of a channel $i, R_{k}$ is the noise ratio $\left(R_{1}=5 \%, \ldots, R_{20}\right.$ $=100 \%), s\left(x_{t}\right)$ is the mean value of the standard deviations of all normalized channel EEGs at time $t$, and $g_{i}$ is the normalized Gaussian random noise. In constructing the dendrograms from the perturbed EEG data set of all patients, we investigate how much elements of the dendrograms remain with the same clustering structure at the different perturbation levels. For each cluster [one cluster consists of five LTLE patients separated by the threshold in Fig. 3(e), the other cluster consists of the other six patients], a cluster- 

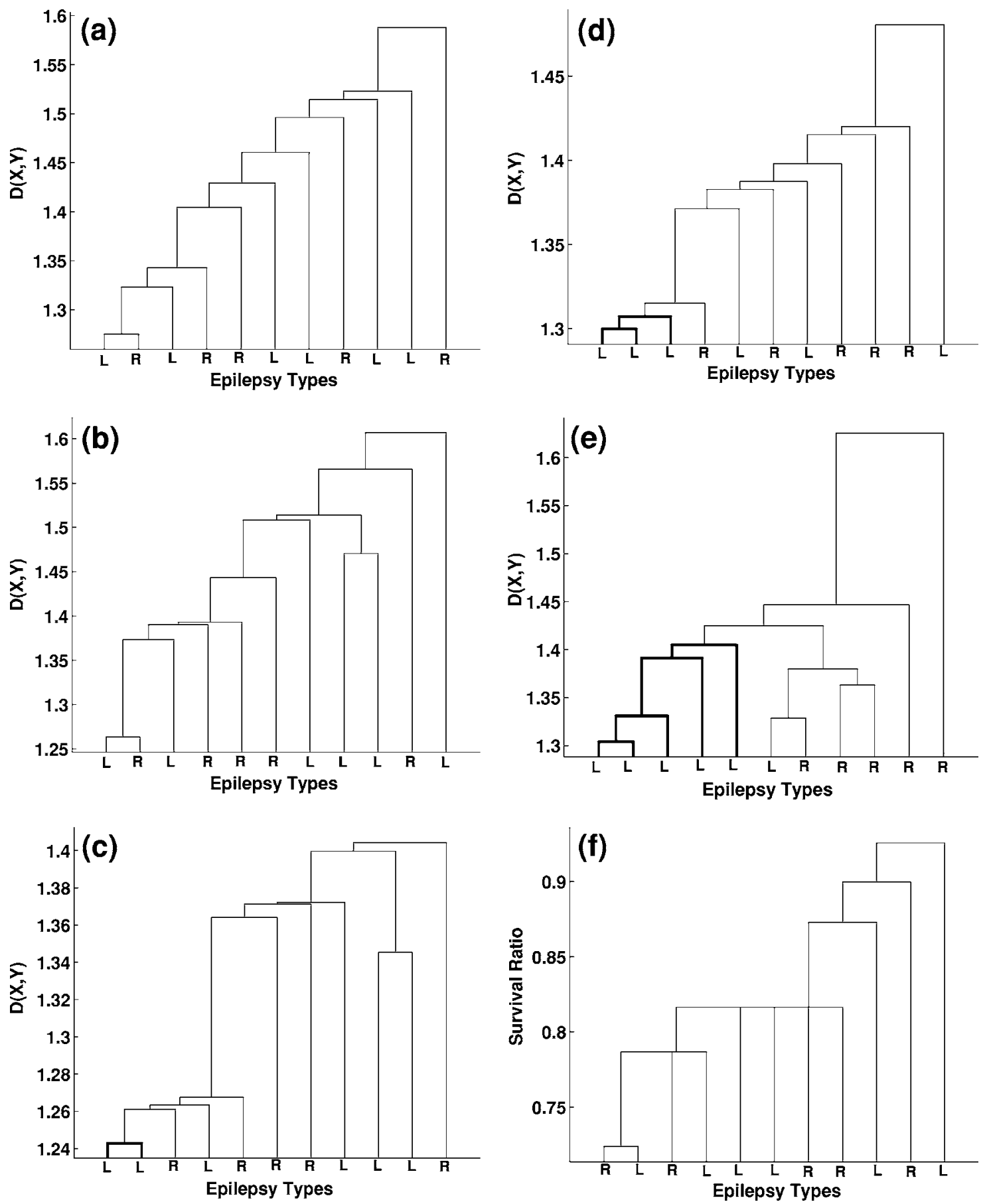

FIG. 3. Dendrograms of the 40 min scalp EEGs are segmented into five EEG periods of 8 min. The dendrograms are constructed with 11 MSTs of all epilepsy patients at each segmented EEG period, respectively. (a)-(e) are dendrograms constructed with $D_{(X, Y)}$ as a measure of dissimilarity. (a) The first EEG epoch (from 32 to $40 \mathrm{~min}$ ), (b) the second EEG epoch (from 24 to 32 min), (c) the third EEG epoch (from 16 to $24 \mathrm{~min}$ ), (d) the fourth EEG epoch (from 8 to $16 \mathrm{~min}$ ), (e) the fifth EEG epoch (from 0 to 8 min before seizure onset), and (f) the dendrogram constructed with the survival ratio as a measure of dissimilarity, at the fifth EEG epoch.

specific discrepancy rate is calculated as $D / M$, where $D$ is the number of patient pairs that do not remain together in the clustering of the perturbed data and $M$ is the number of patient pairs in the original cluster. We found that $D / M$ at all perturbation levels is zero, that is, two clusters remain without exchanging their elements in all perturbation levels. In Fig. 5, we present the discriminative weight (DW) of two types of epilepsy for the divergence rate and the survival ratio. Theoretically, if DW is less than 1, it indicates the two types of epilepsy have no discriminative capability [26,27]. In comparison of two dissimilarity measures, the divergence rate is more discriminative than the survival ratio at all perturbation levels, and the DW is not so sensitive to the perturbation of random noise. Therefore, from this perturbation test, we can show that the hierarchical clustering of two types of epilepsy in Fig. 3(e) is robust and the divergence 

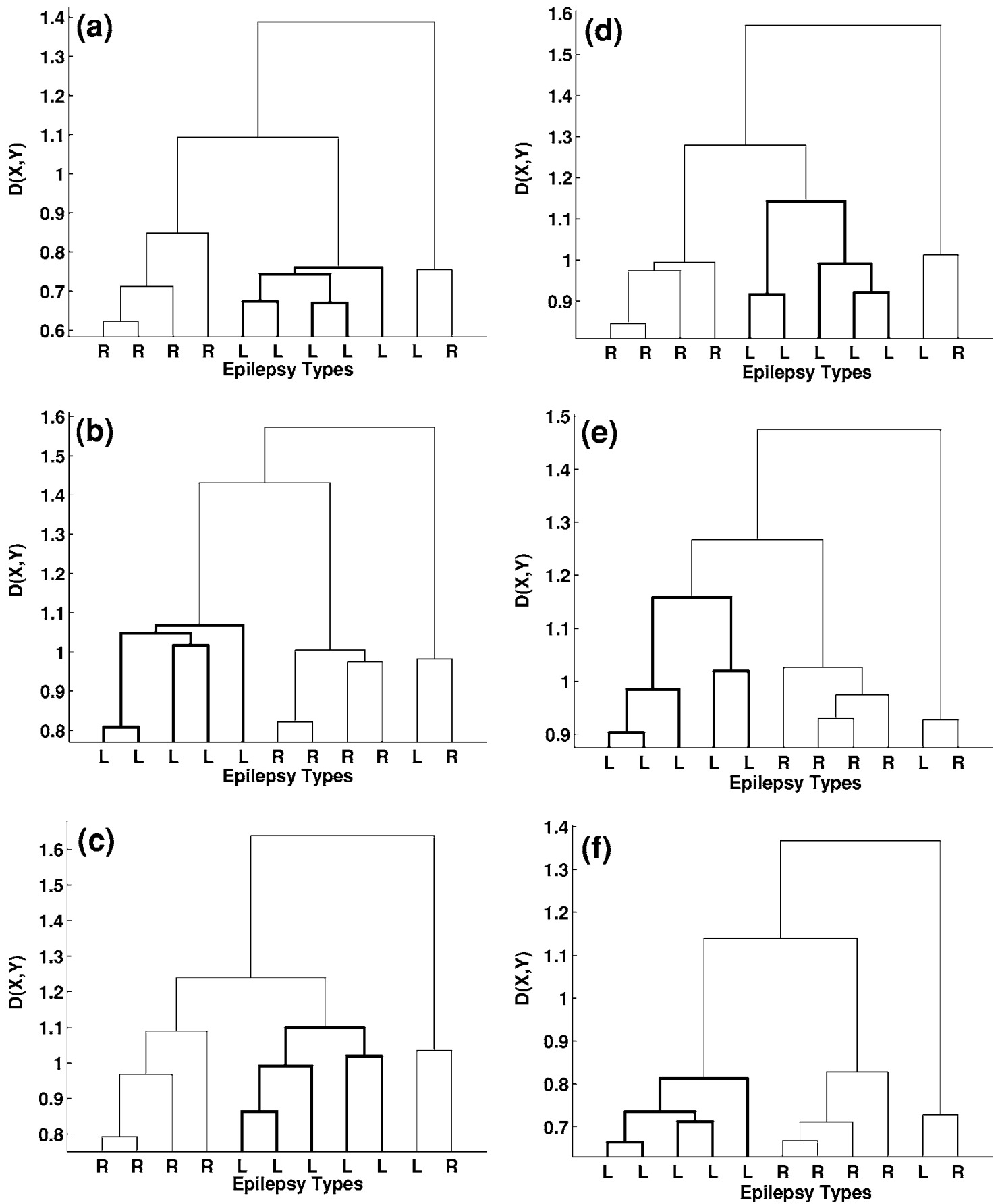

FIG. 4. (a)-(f) Dendrograms of the fifth EEG epoch segmented into six EEG periods of $10 \mathrm{~s}$. Each dendrogram is constructed on the segmented EEG duration which begins at (a) 60, (b) 50, (c) 40, (d) 30, (e) 20, and (f) $10 \mathrm{~s}$ before the seizure onset.

rate is appropriate as a dissimilarity measure of the hierarchical networks.

\section{CONCLUSION}

In this paper, we hypothesized that different types of epilepsy have different encephalographic network structures. To test the difference between both types of epilepsy, we used a repeated measures ANOVA test with the mean cross correlations of all epilepsy patients. This test showed that the dif- ference in both types of epilepsy is not significant. We also investigated the global feature of the channel relationship in multichannel EEGs. We used the MST to construct the hierarchical network of multichannel EEGs, reducing the size of the correlation matrix, while maintaining the skeleton of the interrelationship. The dissimilarity of two network structures is quantified by defining $D_{(X, Y)}$, which measures both the linkage of nodes and the distance of edges. We found that in comparison with the survival ratio $D_{(X, Y)}$ can serve as a good measure for clustering the network structures constructed 


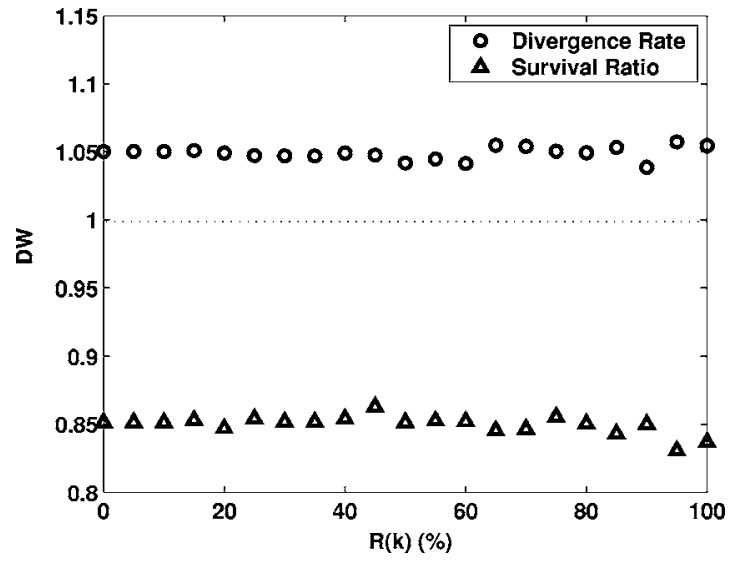

FIG. 5. The discriminative weights (DWs) of two epilepsy types based on two different dissimilarity measures are presented at different perturbation levels. The perturbation level $R(k)$ is given from $5 \%$ to $100 \%$ in proportion to the mean standard deviation of all normalized channel EEGs.

from the scalp EEGs of epilepsy patients, reflecting the contraction or expansion of local subtrees occurring in the epileptic process.

The dendrogram technique based on the $D_{(X, Y)}$ as a dissimilarity measure is found to classify two types of epilepsy, LTLE and RTLE, which exhibit different network structures. In particular, as the seizure onset is approached the network structures of epilepsy patients are found to become more and more distinct. Such an emergence of similar network struc- tures within a group could be understood as evidence of the presence of latent networks, which may be associated with facilitation of the seizure onset. To test the robustness of the classification result, we segmented the 1 min EEG period into $10 \mathrm{~s}$ EEG intervals. We found that the two types of epilepsy are also well classified in the 10 s EEG time scales. We also applied the perturbation test with the WADP method, adding a Gaussian random noise. We found that the classification result and the hierarchical network structure of multichannel EEGs are not sensitive to the perturbation of random noise, ensuring the robustness of this methodology based on the divergence rate.

Our results suggest that the global features of the channel relationship based on MST and their time evolution provide meaningful information on the transient brain state of an epilepsy patient. Our approach could be used for classifying other epilepsy types or monitoring the change of the dynamical state of the epilepsy patient. Further work on the global network on a specific spectral band, which uses phase synchronization instead of cross correlation, and the dependence of the global network structure on the EEG time scale is needed.

\section{ACKNOWLEDGMENTS}

This work has been supported by the Ministry of Science \& Technology through the National Research Laboratory Project and the National Core Research Center on System Bio-Dynamics.
[1] K. Lehnertz, Int. J. Psychophysiol 34, 45 (1999).

[2] J. Arnhold, P. Grassberger, K. Lehnertz, and C. E. Elger, Physica D 134, 419 (1999).

[3] M. Le Van Quyen, C. Adam, M. Baulac, J. Martinerie, and F. Varela, Brain Res. 792, 24 (1998)

[4] M. Le Van Quyen, J. Martinerie, C. Adam, and F. Varela, Physica D 127, 250 (1999)

[5] F. Mormann, K. Lehnertz, P. David, and C. E. Elger, Physica D 144, 358 (2000)

[6] F. Mormann, T. Kreuz, R. G. Andrzejak, P. David, K. Lehnertz, and C. E. Elger, Epilepsy Res. 53, 173 (2003)

[7] E. Rodriguez, Nature (London) 397, 430 (1999)

[8] F. Varela, J.-P. Lachaux, E. Rodriguez, and J. Martinerie, Nat. Rev. Neurosci. 2, 229 (2001)

[9] A. Lutz, Phenom. Cogn. Sci. 1, 133 (2002)

[10] M. Le Van Quyen and C. Petitmengin, Phenom. Cogn. Sci. 1, 169 (2002)

[11] L. S. Boylan, Epilepsy Behavior 3, 16 (2002)

[12] P. Blanchet and G. P. Frommer, SIGACT News 174, 471 (1986)

[13] J. P. Onnela, A. Chakraborti, K. Kaski, and J. Kertesz, Eur. Phys. J. B 30, 285 (2002)

[14] J. P. Onnela, A. Chakraborti, K. Kaski, and J. Kertesz, Physica A 324, 247 (2003)

[15] J. P. Onnela, K. Kaski, and J. Kertesz, Eur. Phys. J. B 38, 353 (2004)
[16] R. N. Mantegna, Eur. Phys. J. B 11, 193 (1999)

[17] G. Bonanno, N. Vandewalle, and R. N. Mantegna, Phys. Rev. E 62, R7615 (2000)

[18] R. Rammal, G. Toulouse, and M. A. Virasoro, Rev. Mod. Phys. 58, 765 (1986)

[19] L. Lam and H. C. Morris, Nonlinear Structure in Physical Systems-Pattern Formation, Chaos and Waves (SpringerVerlag, New York, 1990).

[20] B. Everitt, Cluster Analysis (Heinemann, London, 1974).

[21] M. Le Van Quyen, J. Martinerie, M. Baulac, and F. Varela, NeuroReport 10, 2149 (1999)

[22] M. Le Van Quyen, V. Navarro, M. Baulac, B. Renault, and J. Martinerie, Lancet 361, 970 (2003)

[23] V. Navarro, Brain 125, 640 (2002)

[24] F. Mormann, R. G. Andrzejak, T. Kreuz, C. Rieke, P. David, C. E. Elger, and K. Lehnertz, Phys. Rev. E 67, 021912 (2003)

[25] M. Bittner, et al., Nature (London) 406, 6795 (2000)

[26] The DW is defines as $d_{B} /\left(k_{1} d w_{1}+k_{2} d w_{2}+\alpha\right)$, where $d_{B}$ is the center-to-center distance (between-cluster Euclidean distance), $d w_{i}$ is the average Euclidean distance among all sample pairs within cluster $i$, and $k_{i}=t_{i} /\left(t_{1}+t_{2}\right)$ is the weight for the number of samples in the cluster, and $\alpha$ is a small constant (0.01) to prevent the zero-dominator case.

[27] B. Everitt, Applied Multivariate Data Analysis (Oxford University Press, New York, 1992). 